\title{
Identification of hub genes related to silicone-induced immune response in rats
}

\author{
Xiaolu Huang ${ }^{1, *}$, Yiwen Zhou ${ }^{1, *}$, Wenhui Liu ${ }^{1}$, Haizhou Li ${ }^{1}$, Xiao Liang ${ }^{1}$, Rui Jin ${ }^{1}$, \\ Hengyu Du ${ }^{1}$, Jizhou He ${ }^{1}$, Bangda Chai ${ }^{1}$, Ran Duan ${ }^{1}$ and Qingfeng $\mathbf{L i}^{1}$ \\ ${ }^{1}$ Department of Plastic and Reconstructive Surgery, Shanghai Ninth People's Hospital, Shanghai Jiao Tong University School \\ of Medicine, Shanghai 200011, P.R.China \\ *These authors contributed equally to this work and should be considered as co-author
}

Correspondence to: Qingfeng Li, email: dr.liqingfeng@shsmu.edu.cn

Keywords: silicone implant, autoimmunity, microarray, WGCNA, hub genes

Received: July 04, 2017 Accepted: September 21, $2017 \quad$ Published: October 06, 2017

Copyright: Huang et al. This is an open-access article distributed under the terms of the Creative Commons Attribution License 3.0 (CC BY 3.0), which permits unrestricted use, distribution, and reproduction in any medium, provided the original author and source are credited.

\section{ABSTRACT}

Silicone implants are used widely in the field of plastic surgery and are used in a large population. However, their safety profile, especially the silicone-induced immune response, has been a major concern for plastic surgeons for decades. It has been hypothesized that there is a cause and effect relation between silicone and immunity, but this is controversial. The objective of the present study was to determine the hub genes and key pathways related to silicone implant-induced immune responses in a rat model. In addition to cluster and enrichment analyses, we used weighted gene co-expression network analysis (WGCNA) to examine the gene expression profiles in a systematic context. A total five genes (Fes, Aif1, Gata3, TIr6, TIr2) were identified as hub genes that are most likely related to the silicone-induced immune response, four of which (Aif1, Gata3, TIr6, TIr2) have been associated with autoimmunity as target genes or disease markers. The Toll-like receptor signaling pathway $(p<0.01$, fold enrichment: 7.01) and systemic lupus erythematosus signaling pathway $(p<0.05$, fold enrichment: 5.01), which are considered strongly associated with autoimmunity, were significantly enriched in the silicone-implanted skin samples. The results indicate that silicone implants might trigger the localized immune response, as various immune reaction genes were detected after silicone implantation. The identified five hub genes will hopefully serve as novel therapeutic targets for silicone-related complications and the associated autoimmune diseases.

\section{INTRODUCTION}

In past decades, millions of people have been exposed to silicone under different circumstances, especially in the area of plastic and reconstructive surgery, where silicone implants are considered the most popular candidate tools for augmentation (e.g. breast implants, tissue expanders, nasal prostheses). Given the wide application and large population exposure, the safety profile of silicone implantation has become a major public health concern.

Apart from common silicone-induced complications (capsule formation and contracture), the fact that silicone implants can increase the risk of connective tissue disease or even autoimmunity has aroused great interest from both researchers and doctors. The US Food and Drug Administration (FDA) limited the use of siliconefilled breast implants in 1992 due to safety concerns [1]. Although the FDA finally reversed its decision for lack of robust evidence, the controversy around this issue has never abated. The evidence-based meta-analysis by Janowsky et al. [2] concluded that the adjusted relative risk between breast implants and connective tissue disease was 0.8 (95\% confidence interval [CI]: 0.62-1.04). The authors reached this negative conclusion without including some strongly positive population-based cohort studies [3], which therefore prompted some queries [4]. 
On the other hand, some large-scale populationbased epidemiological investigations have verified the postulated association between silicone implants and autoimmunity [5-7]. The improvement of clinical manifestations after implant removal further supports the relationship between silicone and autoimmunity [8]. Though Cohen Tervaert JW et al. has demonstrated that silicone implants could activate around inflammatory cells which contributes to an autoimmune condition [9], the underlying molecular mechanism of this development and progression has been rarely addressed.

Hence, we used dynamic microarray expression datasets and performed a primary investigation aiming to reveal the hub genes (the topmost interconnected genes that are considered to be the backbones of the coexpression networks $[10,11])$ and key pathways involved in the silicone implant-induced local immune response. Comprehensive bioinformatics analyses were used to enrich datasets for Gene Ontology (GO) and pathway information to provide deeper insight into the biological process of the local immune response after silicone implantation. Furthermore, we used dynamic co-expression network construction (similar patterns of connection strengths) and gene connectivity detection to predict the hub genes most likely to contribute to the silicone-induced immune response. A total five genes (Fes, Aif1, Gata3, Tlr6, Tlr2) and nine pathways were identified as central participants in the silicone-induced immune response, most of which are also related to autoimmunity. These genes and pathways will hopefully serve as novel therapeutic targets for silicone-related complications and associated diseases.

\section{RESULTS}

\section{Cluster analysis of significant differential genes}

A total 5,587 genes were identified as differentially expressed $(p<0.05)$, and 1,013 genes were included in significant model patterns (Figure 1), of which 117 genes were enriched in immune response (GO:0006955, $p=2.5 \mathrm{E}-11$, Supplementary Table 1). When clusters and then profiles were ordered based on actual size and the $p$-value of gene enrichment in immune response (GO:0006955), eight profiles had a significant model pattern and five clusters were significantly enriched in the target GO term. Followed by intersection analysis, two profiles (80 genes) were statistically significant for both expression pattern and GO term enrichment (Figure 2); the expression information of these genes is detailed in Supplementary Table 2.

\section{GO analysis based on cluster analysis}

GO analysis was used to identify a subset of the differentially expressed genes corresponding to immune response. A total 80 genes from cluster analysis were assigned to GO terms (Supplementary Tables 1, 2). After filtering using the significant criterion of corrected $p<0.05$, we selected $24 \mathrm{GO}$ terms with key functional classifications (Table 1).

An interaction network of significant GO terms was assembled into a GO map using ClueGO to depict the relationship among prominent functional categories (Supplementary Figure 1). Based on the GO map, we were able to directly and systematically find the subordinate relationship between GO terms. Comparison of the comprehensive $\mathrm{GO}$ analysis and cluster $\mathrm{GO}$ analysis suggested that immune response, leukocyte activation, lymphocyte activation and immune effector process, and signaling processes were the most prominent functions after silicone implantation.

\section{Pathway analysis}

We used DAVID software based on the KEGG pathway map to investigate key pathways linked to the 80 genes. Our analysis yielded nine statistically significant pathways (Table 2) involving hematopoietic cell lineage, Toll-like receptor (TLR) signaling, and NOD-like receptor signaling. The association between autoimmunity and silicone implantation was highlighted by the identification of the systemic lupus erythematosus (SLE) signaling pathway (Figure 3, top) and TLR signaling pathway (Figure 3, bottom).

\section{WGCNA and hub gene detection}

We selected 1,013 genes for further analysis by constructing a weighted gene co-expression network. First, we identified six network modules, which are illustrated in the dendrogram (Supplementary Figure 2). In network terminology, modules are groups of genes with similar patterns of connection strengths with all other genes in the network and they usually share similar functions [12].

After module detection, the connection strength between two genes was determined using soft thresholding of the Pearson correlation matrix [12]. Figure 4 demonstrates the co-expression network of the 80 selected genes, which reflects the correlations between the genes. Each node describes a given gene, and the relationship between a pair of genes is represented with an edge. Further, the area of the node represents its k-core value within the module, and the edge correlates with the capacity for modulating adjacent genes. Genes with higher k-core values are more centralized in the network and have a stronger capacity for modulating adjacent genes. Consequently, we identified five genes with the highest k-core value in the network as hub genes in the immune response after silicone implantation, namely, Fes, Aif1, Tlr6, Tlr2, and Gata3. Twenty-five genes with the highest k-core values were subsequently assigned to significant GO terms to investigate the distribution of their participation; Figure 5 depicts the heatmap. 
Table 1: The summary of GO terms in significant expression patterns profiles

\begin{tabular}{|c|c|c|c|c|c|c|c|c|}
\hline Category ID & Category Name & $\begin{array}{c}\text { Genes } \\
\text { Category }\end{array}$ & $\begin{array}{c}\text { Genes } \\
\text { Assigned }\end{array}$ & $\begin{array}{c}\text { Genes } \\
\text { Expected }\end{array}$ & $\begin{array}{c}\text { Genes } \\
\text { Enriched }\end{array}$ & $p$-value & $\begin{array}{c}\text { Corrected } \\
p \text {-value }\end{array}$ & Fold \\
\hline \multicolumn{9}{|l|}{ Profile 38} \\
\hline GO:0006955 & immune response & 396 & 49 & 17.8 & 31.2 & $2.50 \mathrm{E}-11$ & $<0.001$ & 2.7 \\
\hline GO:0045321 & leukocyte activation & 286 & 35 & 12.9 & 22.1 & $3.60 \mathrm{E}-08$ & $<0.001$ & 2.7 \\
\hline GO:0009617 & response to bacterium & 275 & 34 & 12.4 & 21.6 & 4.40E-08 & $<0.001$ & 2.7 \\
\hline GO:0009611 & response to wounding & 476 & 48 & 21.4 & 26.6 & $4.50 \mathrm{E}-08$ & $<0.001$ & 2.2 \\
\hline GO:0002252 & immune effector process & 226 & 30 & 10.2 & 19.8 & $6.00 \mathrm{E}-08$ & $<0.001$ & 2.9 \\
\hline GO:0002682 & regulation of immune system process & 401 & 42 & 18.1 & 23.9 & $1.30 \mathrm{E}-07$ & 0.002 & 2.3 \\
\hline GO:0050865 & regulation of cell activation & 212 & 28 & 9.5 & 18.5 & $1.90 \mathrm{E}-07$ & 0.002 & 2.9 \\
\hline GO:0002237 & response to molecule of bacterial origin & 230 & 29 & 10.4 & 18.6 & $3.10 \mathrm{E}-07$ & 0.002 & 2.8 \\
\hline GO:0032101 & regulation of response to external stimulus & 248 & 30 & 11.2 & 18.8 & 4.80E-07 & 0.002 & 2.7 \\
\hline GO:0046649 & lymphocyte activation & 238 & 29 & 10.7 & 18.3 & $6.50 \mathrm{E}-07$ & 0.004 & 2.7 \\
\hline GO:0032496 & response to lipopolysaccharide & 221 & 27 & 10 & 17 & $1.60 \mathrm{E}-06$ & 0.004 & 2.7 \\
\hline GO:0006954 & inflammatory response & 271 & 30 & 12.2 & 17.8 & $3.20 \mathrm{E}-06$ & 0.004 & 2.5 \\
\hline GO:0002366 & leukocyte activation involved in immune response & 76 & 14 & 3.4 & 10.6 & $5.50 \mathrm{E}-06$ & 0.01 & 4.1 \\
\hline GO:0002263 & cell activation involved in immune response & 76 & 14 & 3.4 & 10.6 & $5.50 \mathrm{E}-06$ & 0.01 & 4.1 \\
\hline GO:0002694 & regulation of leukocyte activation & 189 & 23 & 8.5 & 14.5 & $1.00 \mathrm{E}-05$ & 0.022 & 2.7 \\
\hline GO:0051249 & regulation of lymphocyte activation & 163 & 21 & 7.3 & 13.7 & $1.10 \mathrm{E}-05$ & 0.022 & 2.9 \\
\hline GO:0002274 & myeloid leukocyte activation & 75 & 13 & 3.4 & 9.6 & $2.40 \mathrm{E}-05$ & 0.034 & 3.8 \\
\hline \multicolumn{9}{|l|}{ Profile 49} \\
\hline GO:0006955 & immune response & 396 & 31 & 9.8 & 21.2 & 4.70E-09 & $<0.001$ & 3.2 \\
\hline GO:0002682 & regulation of immune system process & 401 & 28 & 10 & 18 & $3.50 \mathrm{E}-07$ & $<0.001$ & 2.8 \\
\hline GO:0006954 & inflammatory response & 271 & 22 & 6.7 & 15.3 & $6.30 \mathrm{E}-07$ & $<0.001$ & 3.3 \\
\hline GO:0002684 & positive regulation of immune system process & 261 & 21 & 6.5 & 14.5 & $1.40 \mathrm{E}-06$ & $<0.001$ & 3.2 \\
\hline GO:0002252 & immune effector process & 226 & 18 & 5.6 & 12.4 & $9.60 \mathrm{E}-06$ & 0.01 & 3.2 \\
\hline GO:0002274 & myeloid leukocyte activation & 75 & 10 & 1.9 & 8.1 & $1.30 \mathrm{E}-05$ & 0.016 & 5.4 \\
\hline GO:0002696 & positive regulation of leukocyte activation & 128 & 13 & 3.2 & 9.8 & $1.40 \mathrm{E}-05$ & 0.018 & 4.1 \\
\hline GO:0002675 & positive regulation of acute inflammatory response & 23 & 6 & 0.6 & 5.4 & $1.50 \mathrm{E}-05$ & 0.024 & 10.5 \\
\hline GO:0030097 & hemopoiesis & 260 & 19 & 6.5 & 12.5 & $1.80 \mathrm{E}-05$ & 0.028 & 2.9 \\
\hline GO:0002823 & $\begin{array}{l}\text { negative regulation of adaptive immune response } \\
\text { based on somatic recombination of immune } \\
\text { receptors built from immunoglobulin superfamily } \\
\text { domains }\end{array}$ & 15 & 5 & 0.4 & 4.6 & $2.20 \mathrm{E}-05$ & 0.04 & 13.4 \\
\hline GO:0002820 & negative regulation of adaptive immune response & 16 & 5 & 0.4 & 4.6 & $3.10 \mathrm{E}-05$ & 0.046 & 12.6 \\
\hline GO:0002697 & regulation of immune effector process & 119 & 12 & 3 & 9 & $3.30 \mathrm{E}-05$ & 0.05 & 4.1 \\
\hline
\end{tabular}

\section{Real-time PCR validation}

The relative expression levels of the five differentially expressed genes (Fes, Aif1, Tlr6, Tlr2, Gata3) were assayed. The results of the microarray assay and RT-PCR were consistent (Supplementary Figure 3).

\section{DISCUSSION}

The present study yields information on the hub genes and key pathways related to the silicone implantinduced immune response as determined using integral bioinformatics methods. We aimed to better understand and characterize local inflammatory and immunologic reactions caused by silicone implants as groundwork to clarify whether this local immunologic reaction can lead to subsequent systemic immune reactions, and even autoimmune diseases. Importantly, by utilizing novel bioinformatics techniques, we bridge the gap between individual genes and systematic biology. The detected hub genes and key pathways are expected to be future therapeutic targets for silicone implant-induced complications and diseases.

Five genes with the highest $\mathrm{k}$-core values were identified as hub genes related to the silicone-induced immune response. Strikingly, most of these hub genes (Tlr2, Tlr6, Aif1, Gata3) have also been reported to be crucial in autoimmunity development. They not only give rise to the localized silicone-induced immune response, but also function as central players in autoimmune disease development [13-18]. 
Table 2: Summary of statistically significant key pathways

\begin{tabular}{|c|c|c|c|c|c|c|}
\hline Term & Genes & Count & $\%$ & $P$-Value & $\begin{array}{c}\text { Fold } \\
\text { Enrichment }\end{array}$ & FDR \\
\hline Cytokine-cytokine receptor interaction & $\begin{array}{l}\text { CSF3, CCL3, TNF, CCL2, CSF1, CXCL2, } \\
\text { CXCL9, TNFSF13, PF4, IL7R, TNFSF18, CCL4, } \\
\text { IL10, CXCL10, OSM, CSF1R }\end{array}$ & 16 & 1.714898 & $1.60 \mathrm{E}-09$ & 7.322744 & $1.73 \mathrm{E}-06$ \\
\hline Hematopoietic cell lineage & $\begin{array}{l}\text { CSF3, CD38, CD55, CD37, TNF, CSF1, FCGR1A, } \\
\text { IL7R, CD14, CSF1R }\end{array}$ & 10 & 1.071811 & $1.21 \mathrm{E}-07$ & 11.55914 & $1.31 \mathrm{E}-04$ \\
\hline Chemokine signaling pathway & $\begin{array}{l}\text { CXCL1, CCL3, CCL2, CXCL2, CXCL9, CCL9, } \\
\text { JAK2, PF4, CCL4, CCL7, CCL6, CXCL10 }\end{array}$ & 12 & 1.286174 & $1.76 \mathrm{E}-06$ & 6.327108 & 0.001906 \\
\hline Toll-like receptor signaling pathway & $\begin{array}{l}\text { CCL3, TNF, TLR2, CXCL9, TLR6, CD14, } \\
\text { CXCL10 }\end{array}$ & 7 & 0.750268 & $4.02 \mathrm{E}-04$ & 7.012545 & 0.433237 \\
\hline NOD-like receptor signaling pathway & CXCL1, TNF, CCL2, CXCL2, CCL7 & 5 & 0.535906 & 0.004465 & 7.271072 & 4.721757 \\
\hline Systemic lupus erythematosus & TNF, FCGR2B, C6, FCGR1A, IL10 & 5 & 0.535906 & 0.01632 & 5.008961 & 16.29149 \\
\hline Jak-STAT signaling pathway & OSM, CSF3, STAT5A, JAK2, IL7R, IL10 & 6 & 0.643087 & 0.017261 & 3.891854 & 17.15306 \\
\hline Natural killer cell mediated cytotoxicity & ITGAL, TNF, FCGR2B, FCER1G, TYROBP & 5 & 0.535906 & 0.023113 & 4.508065 & 22.33229 \\
\hline Asthma & TNF, FCER1G, IL10 & 3 & 0.321543 & 0.025582 & 11.76017 & 24.4271 \\
\hline Fc gamma R-mediated phagocytosis & PTPRC, GAB2, FCGR2B, FCGR1A & 4 & 0.428725 & 0.070663 & 4.09824 & 54.70701 \\
\hline Cell adhesion molecules (CAMs) & ALCAM, ITGAL, PTPRC, CD274, SPN & 5 & 0.535906 & 0.077219 & 3.04599 & 58.04327 \\
\hline Intestinal immune network for $\operatorname{IgA}$ production & TNFSF13, IL10, TGFB1 & 3 & 0.321543 & 0.086023 & 6.010753 & 62.17279 \\
\hline Cytosolic DNA-sensing pathway & IL33, CCL4, CXCL10 & 3 & 0.321543 & 0.089322 & 5.880084 & 63.62241 \\
\hline
\end{tabular}

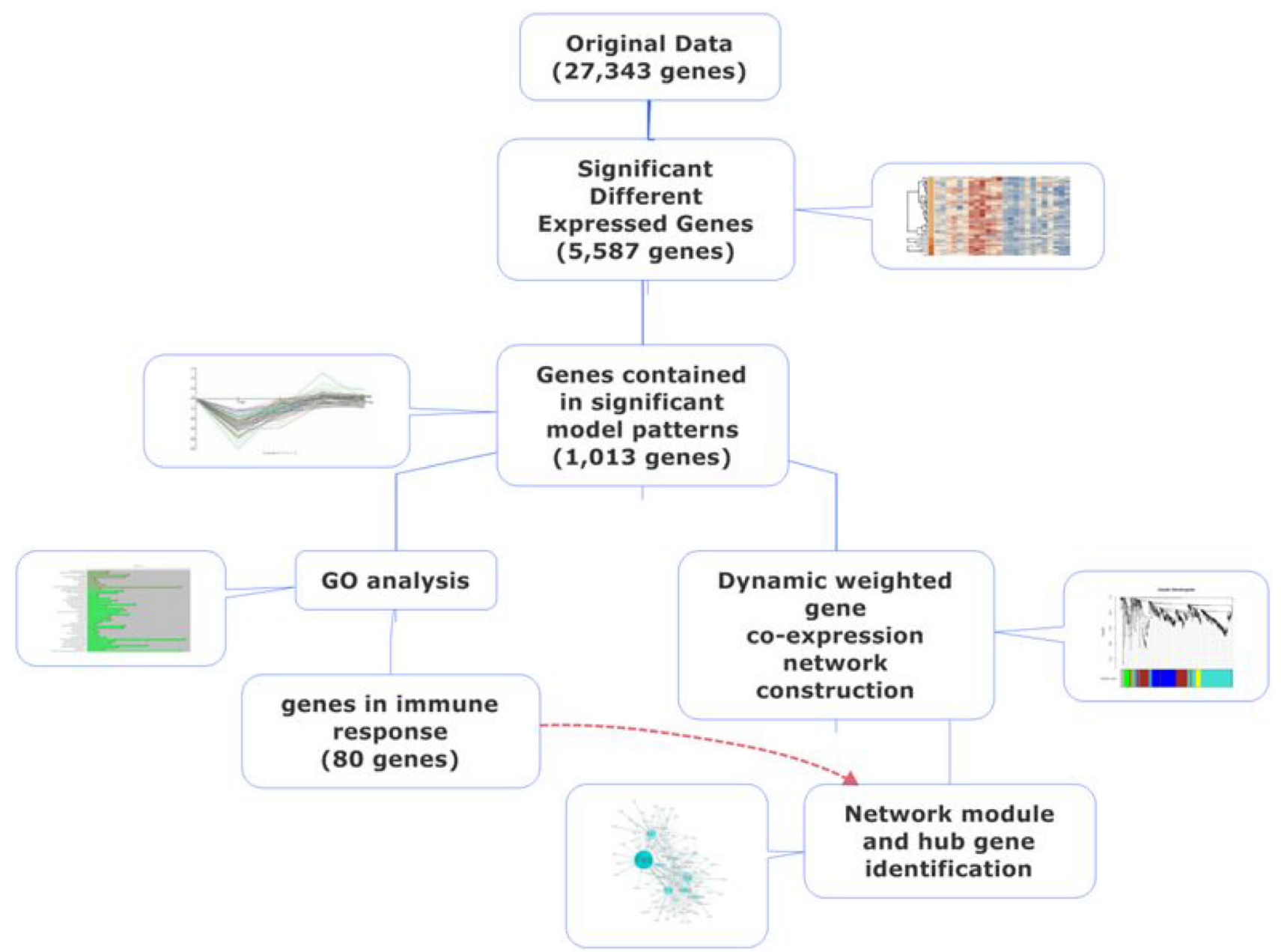

Figure 1: Flow chart of bioinformatics analyses of hub genes and pathways related to immune response after silicone implantation. 
As important components of foreign entity recognition localized on antigen-presenting cells, TLRs are the key signaling molecules in innate immune activation [19]. The downstream activated signaling pathway, termed the TLR signaling pathway (Figure 3, bottom), is the key element of not only adjuvant-induced immune response [5], but also the therapy target in autoimmune disease [19]. In the present study, we found that Tlr2 dimerized with Tlr6 are the major receptors involved in silica surface antigen recognition. Various studies have demonstrated that abnormal and constant activation of TLRs and the TLR signaling pathway can result in sterile inflammation or autoimmunity $[20,21]$ and the subsequent development of a syndrome termed autoimmune syndrome induced by adjuvants (ASIA) [5, 22]. Considering the suggested linkage between autoimmunity and the silicone-induced immune response, novel material should be employed for implantation in the future to avoid activating the $T l r 2 / 6$ signaling pathway to circumvent the common adverse effects stemming from the costly whole-body immune response to antigens.

Allograft inflammatory factor-1 (Aif1), the hub gene with the second highest $\mathrm{k}$-core value, is expressed predominantly by activated monocytes. An increasing number of studies suggest that Aifl may play a critical role in the immune response to allo- or auto-antigens and inflammatory responses [13, 23-24], and its expression level parallels the autoimmune disease stage [25]. Of interest is that, in a rat model with silicone implants, Eltze et al. found a significant correlation between Aifl-positive macrophages and capsule thickness, which indicates that Aif1 might be a novel marker of silicone-induced chronic immune response monitoring [15]. However, the molecular regulatory mechanism of Aifl expression

Profile \#38: $(0,1,2,0,1,0,1)$

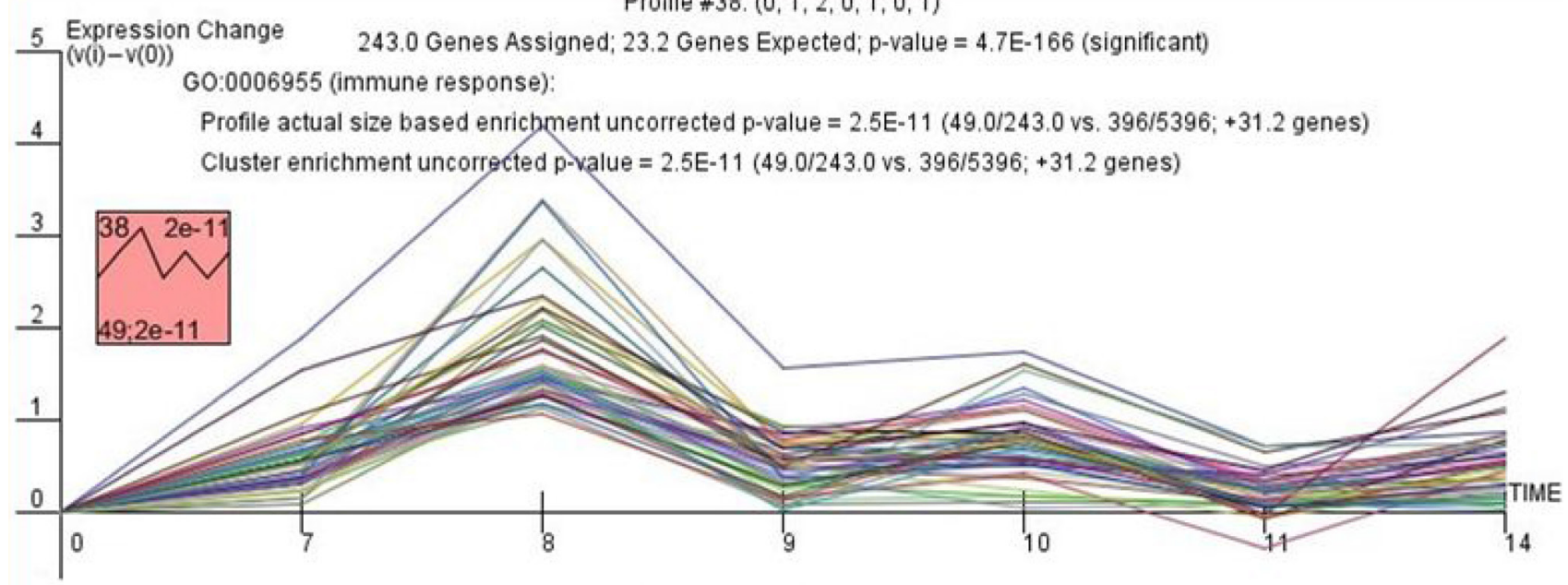

Profile \#49: $(0,2,3,3,3,2,3)$

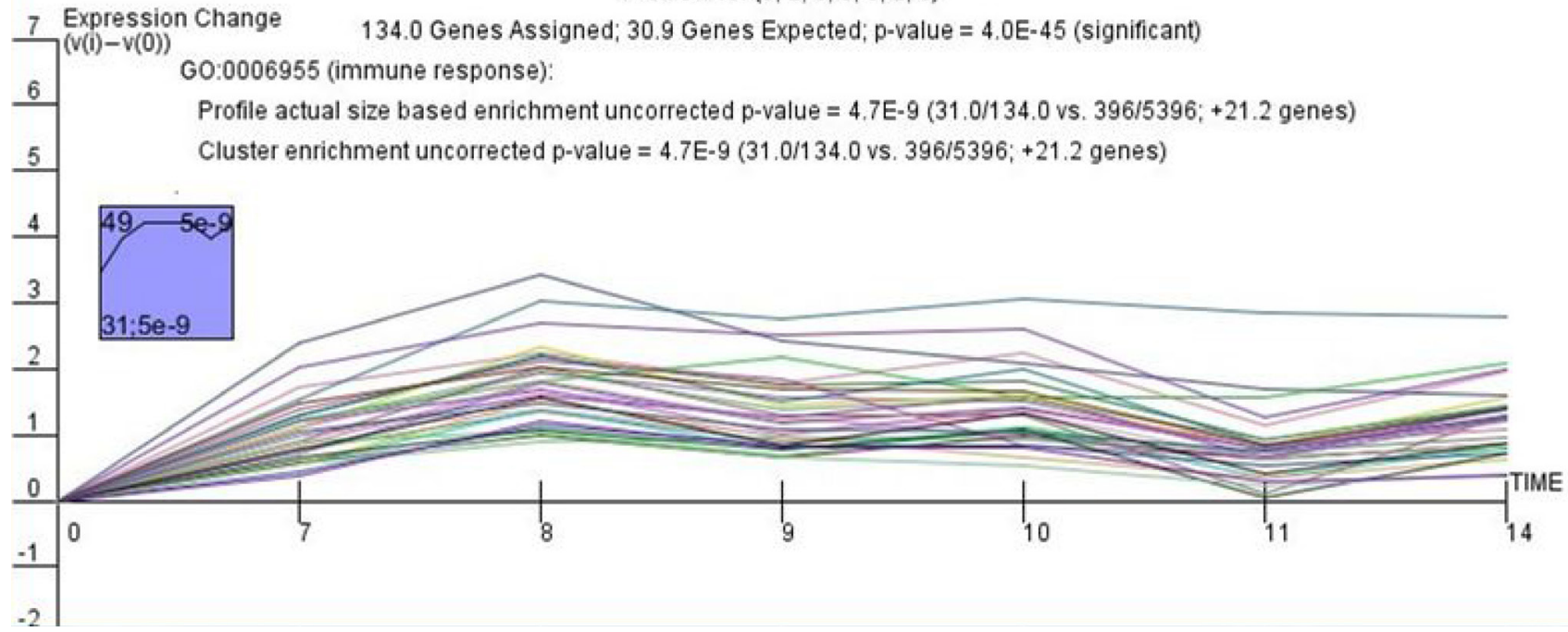

Figure 2: STEM cluster analysis of 80 significantly differential genes after silicone implantation. Two trends $(p<0.001)$ with a statistically significant number of genes were assigned. The boxes on the left side of the figure contain detailed information on these two profiles. In the boxes, the number in the top left corner represents the profile ID, the bottom right corner shows the $p$-value, and the bottom left corner shows the gene number assigned to the profile. Details of the genes mapped to each temporal profile are in Supplementary Table 2. 
and function is unknown. Aifl-1 knockout mice and modern biological technology are important for better understanding of Aifl immune regulatory biology.

The hub gene with the highest $\mathrm{k}$-core value was feline sarcoma oncogene $(\mathrm{Fes})$. However, it is rarely investigated in immunology and little information is currently available to derive preliminary understanding of its function and role in immune-regulation. Further study is necessary to identify specific cell lines that predominantly express $F$ es after silicone implantation and that demonstrate the exact function of Fes when interacting with silicones.
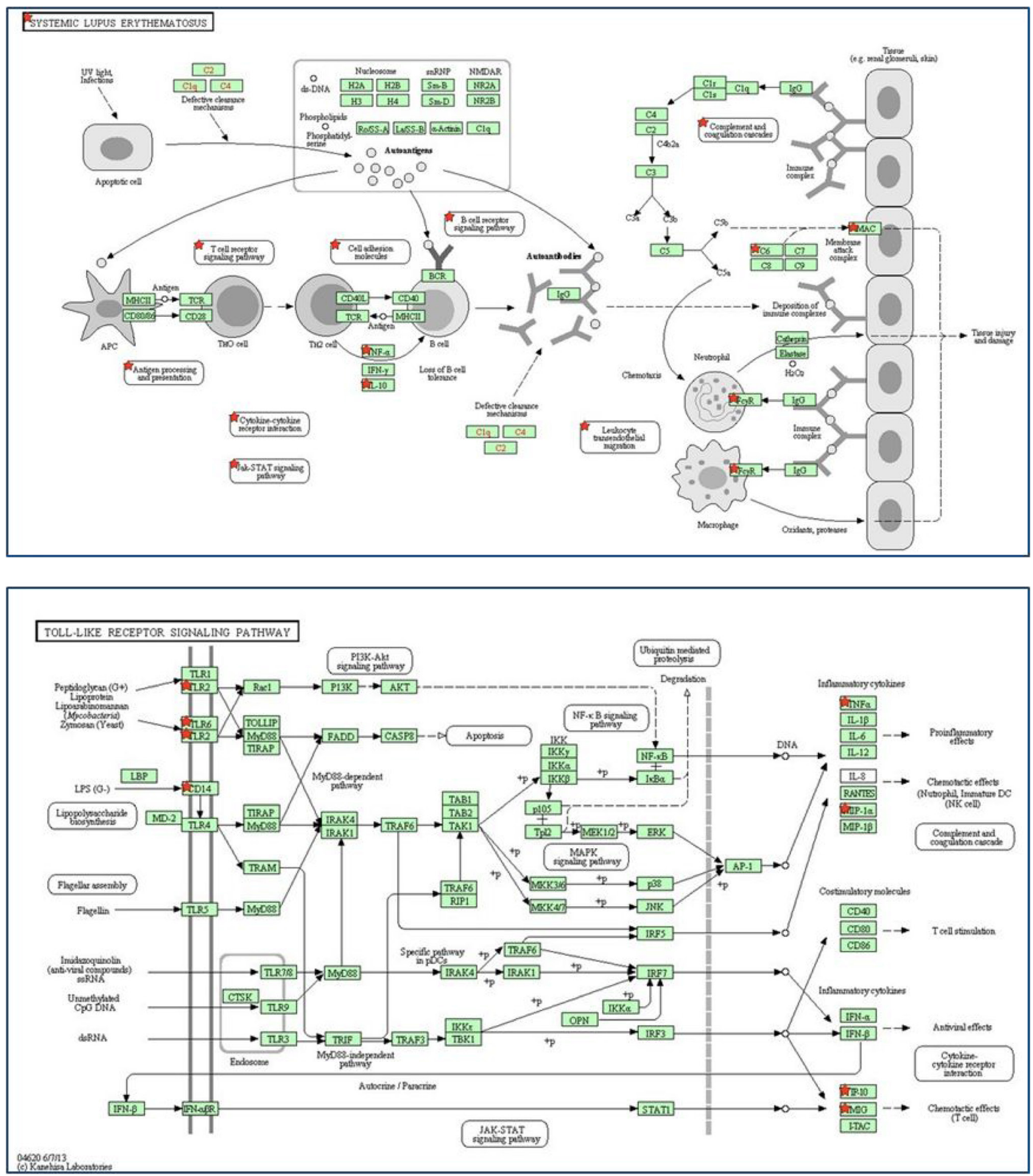

Figure 3: Identification of two autoimmunity-related signaling pathways. Top: SLE signaling pathway; bottom: TLR signaling pathway. Red stars indicate the significantly expressed genes in the pathways. 
The imbalance of $\mathrm{T}$ helper cell (Th1/Th2) differentiation is considered a major pathogenesis step in autoimmune diseases [14, 26, 27]. Interestingly, three of the five hub genes (Gata3, Aif1, Tlr2) function as agonists for Th1 differentiation. Gata3 (GATA Binding Protein 3) plays an indispensable role in Th2 differentiation [28], and its overexpression has been considered a therapeutic target in autoimmune disease [14]. Our data show that
Gata3 had a tendency to be significantly decreased after silicone implantation (Supplementary Table 1, Supplementary Figure 3), indicating imbalanced $\mathrm{T}$ helper cell differentiation that may give rise to Th1 autoimmune disease. Additionally, Aifl regulates Th1 inflammatory responses by augmenting the production of specific cytokines [29], and its expression is increased in Th1-type disease [30, 31]. The characteristic of Tlr2

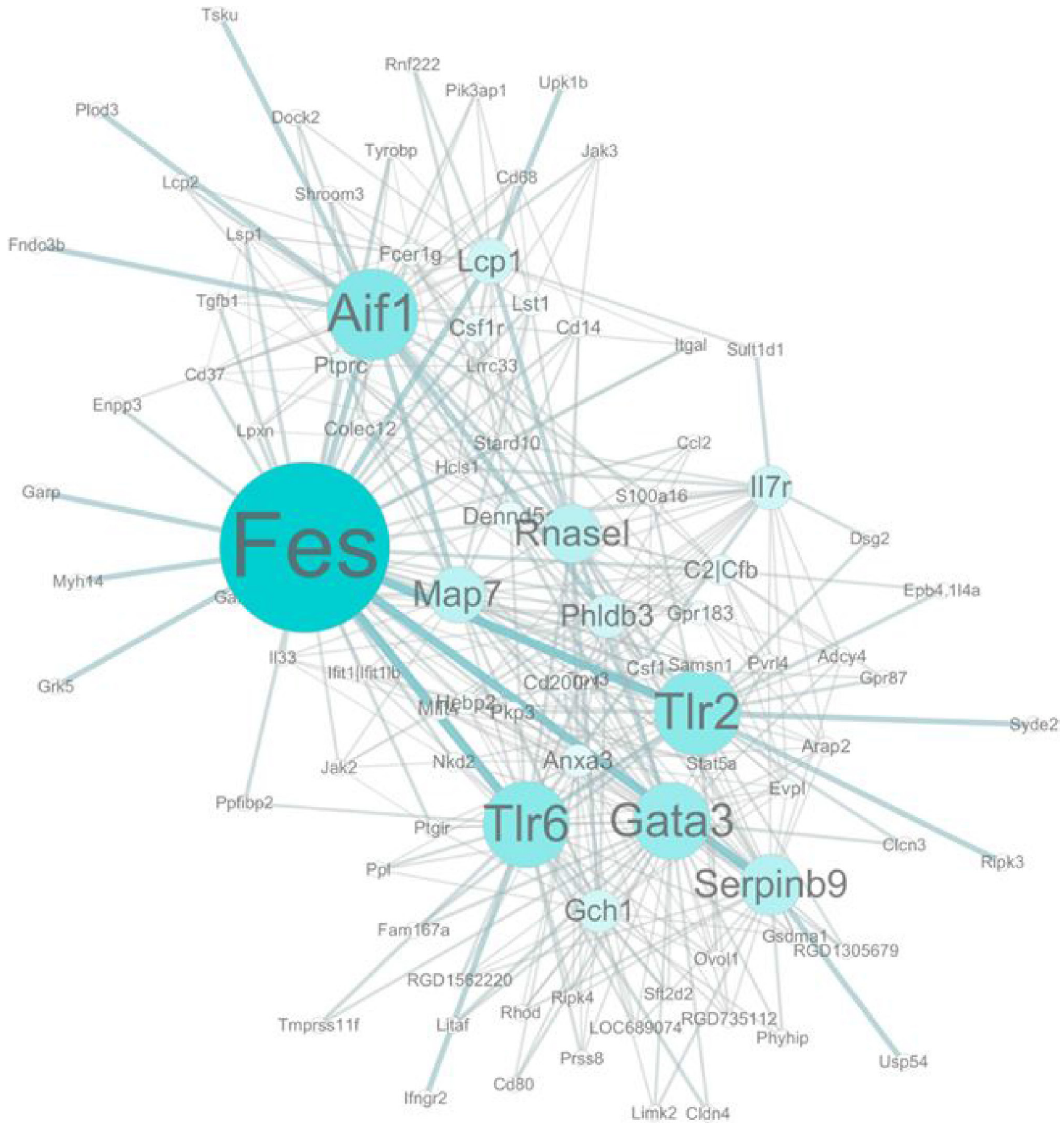

Figure 4: Gene co-expression network. Genes contained in significant GO terms were analyzed and identified by the gene coexpression network using the k-core algorithm. Nodes represent genes; edges indicate the interaction between the genes. The area of each node represents the k-core value within the module, and the edge correlates with the capacity for modulating adjacent genes. Genes with higher k-core values are more centralized in the network and have a stronger capacity for modulating adjacent genes. 
in Th1 differentiation is well-described. Tlr2 is a specific activator of Th1 function and its involvement is implied in Th1-mediated responses [32]. The co-expression of these three genes following silicone implantation suggested abnormally increased differentiation towards Th1 and Th1/Th2 imbalance. However, little is known about how silicone affects $\mathrm{T}$ helper cell differentiation, and our findings might provide clues to the establishment of a novel pathway model for mimicking silicone-induced autoimmunity.

Further investigation will mainly focus on whether interfering with the expression of these hub genes would significantly exacerbate or alleviate the immune response after silicone implantation. Additionally, these data will be further validated and replicated with silicone implants in human samples. The shared target genes and common pathways between the silicone-induced immune response and population-based connective tissue disease microarray data (from the Gene Expression Omnibus [GEO] database) will also be compared and analyzed for further study.

Our study has limitations: the time points span a relatively short period compared to that in human patients, who bear silicone implants for years. However, this setting is based on the principle of co-expression network construction, which mainly depends on relative expression changes between two genes from different samples, rather than the expression level of one single gene [33].
Consequently, the expression level profiles of the single genes we report are of lower reference value than the information on the hub genes, which can only represent the acute immune response triggered by silicone implants. Therefore, the expression results at single gene-level should be interpreted with care (Supplementary Tables 1, 2).

With the integral bioinformatics approach, especially WGCNA, we were able to identify the hub genes of the silicone-induced immune response, and thereby infer its potential relationship with autoimmunity, which is controversial and has perplexed epidemiologists for decades. We hope that the information presented here will prompt not only scientists to develop interference medicines, but also encourage manufacturers to improve silicone surface antigens to elude immune recognition for the purpose of alleviating the suffering of patients with silicone-caused complications and associated diseases.

\section{MATERIALS AND METHODS}

\section{Animals and silicone implantation}

Ethics statement: All animal studies complied with current ethical considerations in Directive 2010/63/EU. We used male specific pathogen-free Sprague-Dawley (SD) rats (8 weeks old; male, average body weight, 250-300 g).

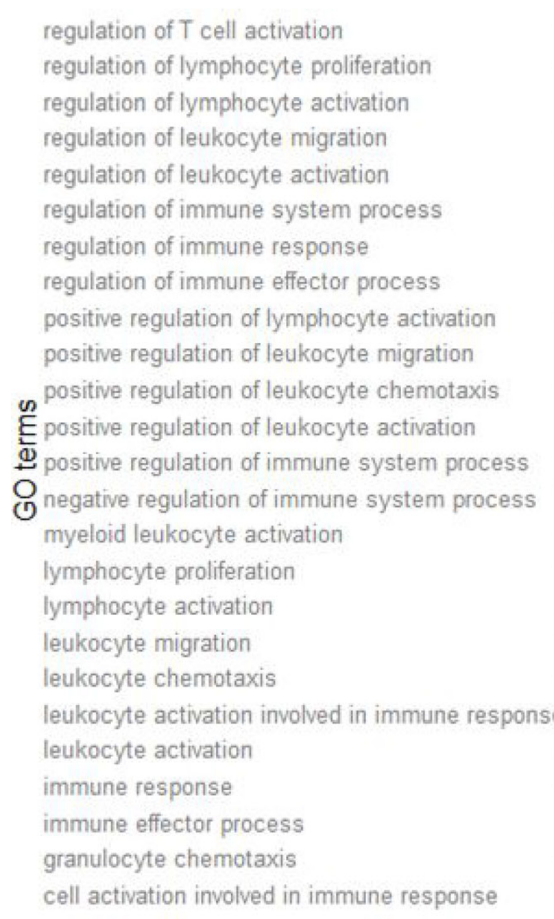

regulation of $T$ cell activation regulation of lymphocyte proliferation regulation of immune system process regulation of immune response regulation of immune effector process positive regulation of leukocyte migration E positive regulation of leukocyte activation $\nsubseteq$ positive regulation of immune system proces: D negative regulation of immune system process myeloid leukocyte activation lymphocyte proliferation lymphocyte activation leukocyte activation involved in immune response leukocyte activation respons granulocyte chemotaxis cell activation involved in immune response

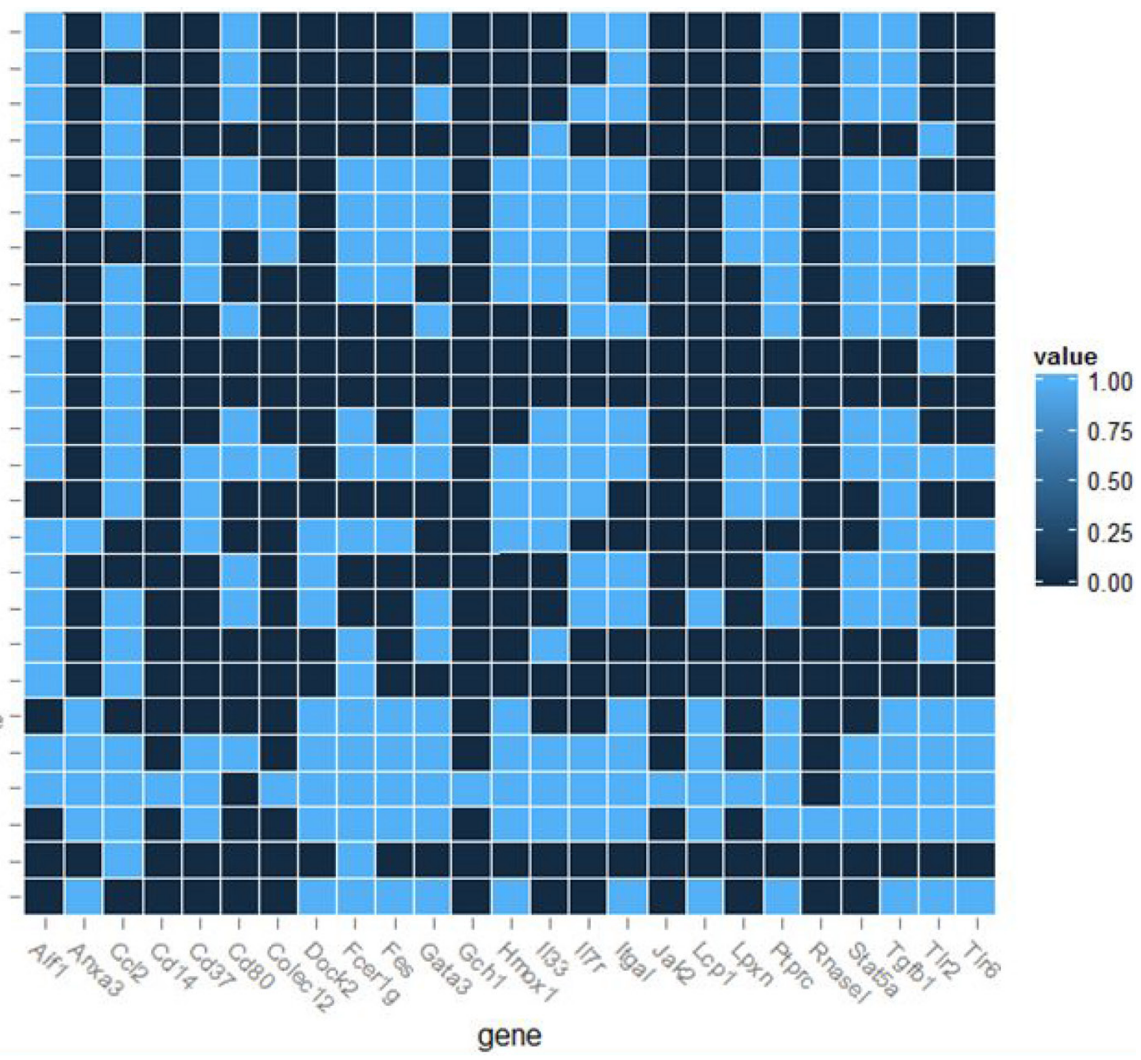

Figure 5: Heat map of distribution of top 25 genes in 25 significant GO terms. The bar on the right indicates if the gene is a participat in the GO term (light blue, yes; dark blue, no). 
The rats were randomly allocated to silicone implantation ( $n=18$, intervention) or sham ( $n=3$, control) groups. in the intervention group were randomly assigned to six time points ( $n=3$ rats per time point): $7,8,9,10,11$, and 14 days. The tissue expander and implantation procedures were described in previous studies $[34,35]$. Briefly, the rats were implanted with $10-\mathrm{ml}$ silicone tissue expanders (silicone MED4735, Shanghai Xinsheng Biomedical Co. Ltd, Shanghai, China; http://www.xinsheng-sh.net/) subcutaneously on the dorsal side (Supplementary Figure 4), and $30 \mathrm{ml}$ saline was injected through the pot to mimic the mechanical stretching after silicone implantation. Rats in the control group underwent a sham procedure without implanting the silicone expanders. At each time point, $0.5 \mathrm{~cm} \times 0.5 \mathrm{~cm}$ full-thickness skin specimens (including the capsule) from the implanted skin area were collected. In the control group, $0.5 \mathrm{~cm} \times 0.5 \mathrm{~cm}$ full-thickness skin specimens from the dorsal midline were collected at day 7. The animals were euthanized immediately after sample collection. The experiments were performed in accordance with the National Experimentation Manual.

\section{RNA isolation}

Total RNA was extracted from the skin specimens, added to TRIzol (Invitrogen, Carlsbad, CA, USA) per the manufacturer's protocol, and was purified using RNeasy Mini columns (Qiagen, Valencia, CA, USA). The absorbance ratio at 260/280 nm (OD 260/280) was measured to evaluate the purity of all RNA samples; RNA quality and integrity were assessed using agarose gel electrophoresis. The $28 \mathrm{~S}$ to $18 \mathrm{~S}$ rRNA band intensity ratio of all RNA samples was approximately $2: 1$; the OD $260 / 280$ ratio was $2.0-2.1$.

\section{Microarray experiment}

RNA samples from all groups were used to detect gene expression changes at each time point. Hybridization, washing, and scanning of Affymetrix GeneChip Rat Exon 1.0 ST Arrays (Santa Clara, CA, USA) were performed according to standard Affymetrix protocols. Statistically significant gene expression was determined using twoway analysis of variance (ANOVA, $p<0.05$ ) because we investigated two factors (silicone implantation and time course). All data generated in this study were minimum information about a microarray experiment (MIAME)compliant [36].

\section{Bioinformatics analysis of microarray data}

Comprehensive bioinformatics analysis was used to determine the hub genes related to the silicone implantationinduced immune response, and included: cluster analysis, GO analysis, pathway analysis, dynamic gene network construction, and hub gene identification (Figure 1).

\section{Cluster analysis}

A total 5,587 significantly expressed genes were assigned to cluster analysis by Short Time-series Expression Miner (STEM) version 1.4 and according to the instructions by Ernst et al. [37]. Fifty model temporal expression patterns were used to identify the expression patterns of the significantly differential genes, and were simultaneously integrated with GO classification. Each cluster contained genes with similar expression patterns. The gene clusters were ranked by the $p$-value of significance of the observed number of genes that fit a profile beyond the expected number.

\section{GO analysis and pathway analysis}

A total 1,013 genes had a significantly differential expression pattern $(p<0.001)$, and underwent GO analysis, which is the key functional classification of the National Center for Biotechnology Information (NCBI) [38]. All GO terms assigned to these genes were obtained, and pathway analysis was enriched using the Database for Annotation, Visualization and Integrated Discovery (DAVID) [39] web server, interrogating the Kyoto Encyclopedia of Genes and Genomes (KEGG) database, and examined simultaneously using Fisher's exact test and the $\chi^{2}$ test for calculating the level of significance. The false discovery rate (FDR) was calculated to correct the $p$-value. Significant genes related to $\mathrm{GO}$ terms named "immune response" were extracted for pattern identification, and the GO term interaction network was visualized using ClueGO [40].

\section{Dynamic weighted gene co-expression network construction}

Next, we used weighted gene co-expression network analysis (WGCNA) to integrate the genes with significant expression patterns into a higher-order, systems-level context [33]. WGCNA is designed to identify modules of densely interconnected genes by searching for genes with similar patterns of connectivity with other genes, which can be summarized as the topological overlap between genes [33]. The blockwiseModules function allowed the entire dataset of 1,013 genes to be utilized in the construction of the weighted gene co-expression network. A pair-wise correlation matrix was computed for each set of genes, and the correlations were weighted to a power of $\beta$ using the power function [33].

\section{Network module and hub gene identification}

The dynamic tree-cutting algorithm [41] was then used to identify the modules of co-expressed genes. After all blocks had been processed, a gene was reassigned to another module if it had higher connectivity to the other 
module, and modules with highly correlated hub genes were merged [42]. Modules, or groups of highly correlated genes, could be a result of transcriptional co-activation (gene activation or gene repression), the co-regulation of mRNA stability, or a combination thereof, resulting in a complex genetic network of closely related genes coordinately operating to accomplish a function or a group of related functions [10].

The TOM function was used to calculate the connectivity (k-core value) of every signal gene in the network; genes with higher k-core values are more centralized in the network and have stronger capacity for modulating adjacent genes. Highly connected "hub" genes are of special interest because they are the backbone of the scale-free network architecture [10], and are far more likely than non-hub genes to be essential for survival in lower organisms [43-45]. As an analogy, the interference of hub genes related to the silicone-induced immune response may lead to amelioration of the pathological process.

\section{Real-time PCR validation of microarray data}

Real-time reverse transcription (RT)-PCR was performed to verify the differential expression of five selected genes: Fes, Aif1, Gata3, Tlr6, and Tlr2. Total RNA was isolated from the skin specimens of rats in the intervention group via microarray assay in three independent experiments. The RNA was reversetranscribed using a ReverTra Ace qPCR RT Kit (TOYOBO, Osaka, Japan). Real-time RT-PCR was conducted in 10-ml reactions consisting of $5 \mathrm{ml}$ SYBR Green Real-time PCR Master Mix (Applied Biosystems), $0.3 \mathrm{mM}$ primers, and $1 \mathrm{ml}$ template complementary DNA (cDNA). The PCR program began with initial denaturation for $5 \mathrm{~min}$ at $94^{\circ} \mathrm{C}$, followed by 40 cycles of $30 \mathrm{sec}$ at $94^{\circ} \mathrm{C}, 30 \mathrm{sec}$ at $60^{\circ} \mathrm{C}$ or $65^{\circ} \mathrm{C}$, and $30 \mathrm{sec}$ at $72^{\circ} \mathrm{C}$, and ended with the melting curve program. The relative changes in gene expression were calculated using the comparative threshold cycle $(2$ $\Delta \Delta \mathrm{Ct})$ method; $\beta$-actin was used as the internal control gene to normalize the amount of RNA used in the PCR [46].

\section{CONCLUSIONS}

The present study results indicate that the siliconeinduced immune response is related to various immune reaction genes and pathways. Five hub genes and nine signaling pathways were identified as central participants in the silicone-induced immune response, most of which are also related to autoimmunity. These genes and pathways will hopefully serve as novel therapeutic targets for silicone-related complications and associated diseases.

\section{Abbreviations}

Fes: Feline sarcoma oncogene; Aif1: Allograft inflammatory factor-1; Tlr6: Toll-like receptor 6; Tlr2:
Toll-like receptor 2; Gata3: GATA Binding Protein 3; ASIA: Autoimmune syndrome induced by adjuvants; DAVID: Database for Annotation, Visualization and Integrated Discovery; FDR: False discovery rate; GEO: Gene Expression Omnibus; GO: Gene ontology; KEGG: Kyoto Encyclopedia of Genes and Genomes; MIAME: Minimum information about a microarray experiment; NCBI: National Center for Biotechnology Information; SLE: Systemic lupus erythematosus; STEM: Short Time-series Expression Miner; TLR: Toll-like receptor; WGCNA: Weighted gene co-expression network analysis.

\section{Author contributions}

Xiaolu Huang developed and executed the idea of identifying hub genes related to silicone immune response, and wrote the manuscript. Yiwen Zhou conducted the RTPCR validation and edited the manuscript. Wenhui Liu and Xiao Liang performed the data processing. Haizhou Li and Hengyu Du helped perform the analysis with constructive discussions. Jizhou He and Ran Duan contributed to the animal model establishing. Rui Jin and Bangda Chai contributed to manuscript revising, and Qingfeng $\mathrm{Li}$ reviewed the original data and approved the manuscript.

\section{ACKNOWLEDGMENTS}

Special thanks to Prof. Yufang Shi for providing great insight for this work. Thanks to all the members in our group that gave us help to complete this work. Thanks to Oxford Science Editing for language support and manuscript editing.

\section{CONFLICTS OF INTEREST}

The authors have no financial interest to declare in relation to the content of this article.

\section{FUNDING}

This work was supported by grants from the National Natural Science Foundation of China (No.81501668, No.81620108019 and No. 81230042).

\section{REFERENCES}

1. Kessler DA. The basis of the FDA's decision on breast implants. N Engl J Med. 1992; 326:1713-5. https://doi. org/10.1056/NEJM199206183262525.

2. Janowsky EC, Kupper LL, Hulka BS. Meta-analyses of the relation between silicone breast implants and the risk of connective-tissue diseases. N Engl J Med. 2000; 342:78190. https://doi.org/10.1056/NEJM200003163421105.

3. Hennekens CH, Lee IM, Cook NR, Hebert PR, Karlson EW, LaMotte F, Manson JE, Buring JE. Self-reported 
breast implants and connective-tissue diseases in female health professionals. A retrospective cohort study. JAMA. 1996; 275:616-21. https://doi.org/10.1001/ jama.1996.03530320040032.

4. Hajdu SD, Agmon-Levin N, Shoenfeld Y. Silicone and autoimmunity. Eur J Clin Invest. 2011; 41:203-11. https:// doi.org/10.1111/j.1365-2362.2010.02389.x.

5. Shoenfeld Y, Agmon-Levin N. 'ASIA' - autoimmune/ inflammatory syndrome induced by adjuvants. J Autoimmun. 2011; 36:4-8. https://doi.org/10.1016/j. jaut.2010.07.003.

6. Watad A, Quaresma M, Brown S, Cohen Tervaert JW, Rodriguez-Pint I, Cervera R, Perricone C, Shoenfeld Y. Autoimmune/inflammatory syndrome induced by adjuvants (Shoenfeld's syndrome) - An update. Lupus. 2017; 26:67581. https://doi.org/10.1177/0961203316686406.

7. Kappel RM, Cohen Tervaert JW, Pruijn GJ. Autoimmune/ inflammatory syndrome induced by adjuvants (ASIA) due to silicone implant incompatibility syndrome in three sisters. Clin Exp Rheumatol. 2014; 32:256-58.

8. Maijers MC, de Blok CJ, Niessen FB, van der Veldt AA, Ritt MJ, Winters HA, Kramer MH, Nanayakkara PW. Women with silicone breast implants and unexplained systemic symptoms: a descriptive cohort study. Neth J Med. 2013; 71:534-40.

9. Cohen Tervaert JW, Colaris MJ, van der Hulst RR. Silicone breast implants and autoimmune rheumatic diseases: myth or reality. Curr Opin Rheumatol. 2017; 29:348-54.https:// doi.org/10.1097/BOR.0000000000000391.

10. Ghazalpour A, Doss S, Zhang B, Wang S, Plaisier C, Castellanos R, Brozell A, Schadt EE, Drake TA, Lusis AJ, Horvath S. Integrating genetic and network analysis to characterize genes related to mouse weight. PLoS Genet. 2006; 2:e130. https://doi.org/10.1371/journal. pgen.0020130.

11. Liu W, Huang X, Liang X, Zhou Y, Li H, Yu Q, Li Q. Identification of Key Modules and Hub Genes of Keloids with Weighted Gene Coexpression Network Analysis. Plast Reconstr Surg. 2017; 139:376-90.https://doi.org/10.1097/ PRS.0000000000003014.

12. Zhang B, Horvath S. A general framework for weighted gene co-expression network analysis. Stat Appl Genet Mol Biol. 2005; 4:Article17. https://doi.org/10.2202/1544 6115.1128.

13. Schluesener HJ, Seid K, Kretzschmar J, Meyermann R. Allograft-inflammatory factor- 1 in rat experimental autoimmune encephalomyelitis, neuritis, and uveitis: expression by activated macrophages and microglial cells. Glia. 1998; 24:244-51.

14. Yoh K, Shibuya K, Morito N, Nakano T, Ishizaki K, Shimohata H, Nose M, Izui S, Shibuya A, Koyama A, Engel JD, Yamamoto M, Takahashi S. Transgenic overexpression of GATA-3 in T lymphocytes improves autoimmune glomerulonephritis in mice with a BXSB/MpJ-Yaa genetic background. J Am Soc Nephrol. 2003; 14:2494-502.
15. Eltze E, Bettendorf O, Rody A, Jackisch C, Herchenröder F, Böcker W, Pfleiderer B. Influence of local complications on capsule formation around model implants in a rat model. J Biomed Mater Res A. 2003; 64:12-19. https://doi. org/10.1002/jbm.a.10342.

16. Eltze E, Schäfer U, Bettendorf O, Rody A, Herchenröder F, Chiwritsch T, Jackisch C, Pfleiderer B. Radiation-induced capsule tissue reactions around textured breast implants in a rat model. Breast. 2006; 15:331-38. https://doi. org/10.1016/j.breast.2005.08.037.

17. Bauer D, Busch M, Pacheco-López G, Kasper M, Wildschütz L, Walscheid K, Bähler H, Schröder M, Thanos S, Schedlowski M, Heiligenhaus A. Behavioral Conditioning of Immune Responses with Cyclosporine A in a Murine Model of Experimental Autoimmune Uveitis. Neuroimmunomodulation. 2017; 24:87-99. https://doi. org/10.1159/000479185.

18. Chen J, Feng X, Huang Q. Modulation of T-Bet and GATA3 expression in experimental autoimmune thyroiditis rats through ginsenoside treatment. Endocr Res. 2016; 41:2833. https://doi.org/10.3109/07435800.2015.1066800.

19. Barrat FJ, Coffman RL. Development of TLR inhibitors for the treatment of autoimmune diseases. Immunol Rev. 2008; 223:271-83. https://doi.org/10.1111/j.1600065X.2008.00630.x.

20. Baccala R, Hoebe K, Kono DH, Beutler B, Theofilopoulos AN. TLR-dependent and TLR-independent pathways of type I interferon induction in systemic autoimmunity. Nat Med. 2007; 13:543-51. https://doi.org/10.1038/nm1590.

21. Marshak-Rothstein A. Toll-like receptors in systemic autoimmune disease. Nat Rev Immunol. 2006; 6:823-35. https://doi.org/10.1038/nri1957.

22. Meroni PL. Autoimmune or auto-inflammatory syndrome induced by adjuvants (ASIA): old truths and a new syndrome? J Autoimmun. 2011; 36:1-3. https://doi. org/10.1016/j.jaut.2010.10.004.

23. Nagakawa $\mathrm{Y}$, Nomoto S, Kato $\mathrm{Y}$, Montgomery RA, Williams GM, Klein AS, Sun Z. Over-expression of AIF-1 in liver allografts and peripheral blood correlates with acute rejection after transplantation in rats. Am J Transplant. 2004; 4:1949-57. https://doi.org/10.1111/ j.1600-6143.2004.00621.x.

24. Beiter T, Artelt MR, Trautmann K, Schluesener HJ. Experimental autoimmune neuritis induces differential microglia activation in the rat spinal cord. $\mathrm{J}$ Neuroimmunol. 2005; 160:25-31. https://doi.org/10.1016/j. jneuroim.2004.10.027.

25. Pashenkov M, Efendic S, Zhu J, Zou LP, Ostenson CG, Mustafa M. Augmented expression of daintain/allograft inflammatory factor-1 is associated with clinical disease: dynamics of daintain/allograft inflammatory factor-1 expression in spleen, peripheral nerves and sera during experimental autoimmune neuritis. Scand J Immunol. 2000; 52:117-22. https://doi.org/10.1046/j.13653083.2000.00682.x. 
26. Davidson A, Diamond B. Autoimmune diseases. N Engl J Med. 2001; 345:340-50. https://doi.org/10.1056/ NEJM200108023450506.

27. Mathisen PM, Tuohy VK. Gene therapy in the treatment of autoimmune disease. Immunol Today. 1998; 19:103-05. https://doi.org/10.1016/S0167-5699(98)80005-4.

28. Rengarajan J, Szabo SJ, Glimcher LH. Transcriptional regulation of Th1/Th2 polarization. Immunol Today. 2000; 21:479-83. https://doi.org/10.1016/S0167-5699(00)01712-6.

29. Liu G, Ma H, Jiang L, Zhao Y. Allograft inflammatory factor-1 and its immune regulation. Autoimmunity. 2007; 40:95-102. https://doi.org/10.1080/08916930601083946.

30. Morohashi $\mathrm{T}$, Iwabuchi $\mathrm{K}$, Watano $\mathrm{K}$, Dashtsoodol N, Mishima T, Nakai Y, Shimada S, Nishida R, Fujii $\mathrm{S}$, Onoé K. Allograft inflammatory factor-1 regulates trinitrobenzene sulphonic acid-induced colitis. Immunology. 2003; 110:112-19. https://doi.org/10.1046/j.13652567.2003.01714.x.

31. Kelemen SE, Autieri MV. Expression of allograft inflammatory factor-1 in $\mathrm{T}$ lymphocytes: a role in T-lymphocyte activation and proliferative arteriopathies. Am J Pathol. 2005; 167:619-26. https://doi.org/10.1016/ S0002-9440(10)63003-9.

32. Imanishi T, Hara H, Suzuki S, Suzuki N, Akira S, Saito T. Cutting edge: TLR2 directly triggers Th1 effector functions. J Immunol. 2007; 178:6715-19.

33. Zhang B, Horvath S. A general framework for weighted gene co-expression network analysis. Stat Appl Genet Mol Biol. 2005; 4:e17. https://doi.org/10.2202/1544-6115.1128.

34. Liang X, Huang X, Zhou Y, Jin R, Li Q. Mechanical Stretching Promotes Skin Tissue Regeneration via Enhancing Mesenchymal Stem Cell Homing and Transdifferentiation. Stem Cells Transl Med. 2016; 5:960 69. https://doi.org/10.5966/sctm.2015-0274.

35. Yang M, Li Q, Sheng L, Li H, Weng R, Zan T. Bone marrow-derived mesenchymal stem cells transplantation accelerates tissue expansion by promoting skin regeneration during expansion. Ann Surg. 2011; 253:202-09. https://doi. org/10.1097/SLA.0b013e3181f9balah.

36. Brazma A, Hingamp P, Quackenbush J, Sherlock G, Spellman P, Stoeckert C, Aach J, Ansorge W, Ball CA, Causton HC, Gaasterland T, Glenisson P, Holstege FC, et al. Minimum information about a microarray experiment (MIAME)-toward standards for microarray data. Nat Genet. 2001; 29:365-71. https://doi.org/10.1038/ng1201-365.
37. Ernst J, Bar-Joseph Z. STEM: a tool for the analysis of short time series gene expression data. BMC Bioinformatics. 2006; 7:191. https://doi.org/10.1186/1471-2105-7-191.

38. Consortium GO, and Gene Ontology Consortium. The gene ontology (GO) project in 2006. Nucleic Acids Res. 2006; 34:D322-26. https://doi.org/10.1093/nar/gkj021.

39. Huang W, Sherman BT, Lempicki RA. Systematic and integrative analysis of large gene lists using DAVID bioinformatics resources. Nat Protoc. 2009; 4:44-57. https://doi.org/10.1038/nprot.2008.211.

40. Bindea G, Mlecnik B, Hackl H, Charoentong P, Tosolini M, Kirilovsky A, Fridman WH, Pagès F, Trajanoski Z, Galon J. ClueGO: a Cytoscape plug-in to decipher functionally grouped gene ontology and pathway annotation networks. Bioinformatics. 2009; 25:1091-93. https://doi.org/10.1093/ bioinformatics/btp101.

41. Langfelder P, Zhang B, Horvath S. Defining clusters from a hierarchical cluster tree: the Dynamic Tree Cut package for R. Bioinformatics. 2008; 24:719-20. https://doi. org/10.1093/bioinformatics/btm563.

42. Langfelder $\mathrm{P}$, Horvath S. WGCNA: an R package for weighted correlation network analysis. BMC Bioinformatics. 2008; 9:559. https://doi.org/10.1186/14712105-9-559.

43. Han JD, Bertin N, Hao T, Goldberg DS, Berriz GF, Zhang LV, Dupuy D, Walhout AJ, Cusick ME, Roth FP, Vidal M. Evidence for dynamically organized modularity in the yeast protein-protein interaction network. Nature. 2004; 430:8893. https://doi.org/10.1038/nature02555.

44. Jeong H, Mason SP, Barabási AL, Oltvai ZN. Lethality and centrality in protein networks. Nature. 2001; 411:41-42. https://doi.org/10.1038/35075138.

45. Carlson MR, Zhang B, Fang Z, Mischel PS, Horvath S, Nelson SF. Gene connectivity, function, and sequence conservation: predictions from modular yeast co-expression networks. BMC Genomics. 2006; 7:40. https://doi. org/10.1186/1471-2164-7-40.

46. Livak KJ, Schmittgen TD. Analysis of relative gene expression data using real-time quantitative PCR and the 2(-Delta Delta C(T)) Method. Methods. 2001; 25:402-08. https://doi.org/10.1006/meth.2001.1262. 